\title{
Crystallizing membrane proteins for structure-function studies using lipidic mesophases
}

\author{
Martin Caffrey ${ }^{1}$ \\ Membrane Structural and Functional Biology Group, School of Biochemistry and Immunology, \\ and School of Medicine, Trinity College Dublin, Dublin 2, Ireland
}

\begin{abstract}
The lipidic cubic phase method for crystallizing membrane proteins has posted some high-profile successes recently. This is especially true in the area of G-protein-coupled receptors, with six new crystallographic structures emerging in the last $3^{1 / 2}$ years. Slowly, it is becoming an accepted method with a proven record and convincing generality. However, it is not a method that is used in every membrane structural biology laboratory and that is unfortunate. The reluctance in adopting it is attributable, in part, to the anticipated difficulties associated with handling the sticky viscous cubic mesophase in which crystals grow. Harvesting and collecting diffraction data with the mesophase-grown crystals is also viewed with some trepidation. It is acknowledged that there are challenges associated with the method. However, over the years, we have worked to make the method user-friendly. To this end, tools for handling the mesophase in the pico- to nano-litre volume range have been developed for efficient crystallization screening in manual and robotic modes. Glass crystallization plates have been built that provide unparalleled optical quality and sensitivity to nascent crystals. Lipid and precipitant screens have been implemented for a more rational approach to crystallogenesis, such that the method can now be applied to a wide variety of membrane protein types and sizes. In the present article, these assorted advances are outlined, along with a summary of the membrane proteins that have yielded to the method. The challenges that must be overcome to develop the method further are described.
\end{abstract}

\section{Keywords}

crystallization; G-protein-coupled receptor (GPCR); lipidic cubic phase; macromolecular crystallography; membrane protein structure; mesophase; robot; structure-function analysis

\section{Introduction}

The 13 January 2011 issue of the journal Nature includes three papers reporting on the structure and function of medically and pharmaceutically important GPCRs (Gproteincoupled receptors) [1-3]. Structures were determined using MX (macromolecular crystallography) and in two of these papers, the lipidic cubic phase method was used to generate diffraction quality crystals. The latter, so-called in meso crystallogenesis method, is the focus of the present article.

The structures described in the Nature papers refer to how the atoms that constitute these receptor molecules are arranged in three-dimensional space. This structural information provides invaluable insight into the detailed workings and interactions of these

\footnotetext{
(C) The Authors Journal compilation (C) 2011 Biochemical Society

${ }^{1}$ To whom correspondence should be addressed (martin.caffrey@tcd.ie).
} 
macromolecules and how they might be regulated for the betterment of humankind. It calls to mind the seminal X-ray work of J.D. Bernal and W.H. Bragg carried out at the University of Cambridge almost a century ago on the structure of the carbon allotropes graphite and diamond, where the relationship between structure and material properties was brought into stark relief. Bernal extended the structure-function principle into the realm of biomacromolecules, contributing in no small way to the development of what we recognize today as Structural Biology [4].

The Membrane Structural and Functional Biology (MS\&FB) group at Trinity College Dublin follows in the Bernal tradition of seeking to decipher and to understand the intricacies of the biomolecular structure-function relationship. Here, the focus is on the biological membrane, a bimolecular lipid leaflet in and on which are situated a multitude of proteins and other molecules. The approach taken in the group is a holistic one in that care is taken to perform measurements and to evaluate results in the context of the native biomembrane. Therefore, for this reason, particular attention is devoted to the role played by lipids in the structure, activity and interactions of membrane proteins, a theme emphasized at this Biochemical Society Annual Symposium.

I began my career working with membrane proteins involved in lipid metabolism and calcium transport $[5,6]$. From early on, I recognized the critical role that lipid-phase properties were likely to play in membrane protein function. The dearth of knowledge in the area at the time induced me to embark on an extended period of investigation devoted almost exclusively to establishing the relationship between lipid molecular structure and mesophase or liquid crystalline behaviour and microstructure [7]. One of the goals of that work was to establish rules for rationally designing lipids of defined functionality. This objective was realized after many years and on the back of a lipid synthesis and mesophase characterization programme established in the MS\&FB group. It has provided the community with a suite of lipids having desirable properties for membrane protein crystallization and other applications (see below).

With a solid training in membrane protein biochemistry and biophysics and a strong background in lipid-phase science my group and I were nicely poised to enter the field of macromolecular crystallography as applied to membrane proteins. The opportunity to do just that, and to repot scientifically, came when E. Landau and J. Rosenbusch at the Biocentre in Basel reported that the light-driven transmembrane proton pump bacteriorhodopsin crystallized in a cubic mesophase when treated with phosphate at molar concentrations [8]. At that time, the group had built up considerable experience working with lipidic mesophases, the cubic phase in particular, and had mapped out the temperature-composition phase diagram for the mono-olein-water system (Figure 1) which was directly relevant to the bacteriorhodopsin crystallogenesis work [8]. Collaboration between the group in Basel and the MS\&FB group was established which led to exchanges of materials, methods, equipment, knowledge and personnel, and to work on the mechanism of-membrane protein crystallization in meso $[9,10]$.

The in meso method was particularly appealing to my aforementioned holistic sensibilities because it offered the prospect that crystallization was taking place from within a lipid bilayer, akin to the native environment encountered in a biomembrane. This was in contrast with the more traditional 'in surfo' methods for crystallizing membrane proteins [7,10a] available at the time which involved using potentially destabilizing surfactant micelles. Altogether, the method and the opportunities it presented made it an attractive direction in which to channel our research efforts which have three major themes. First, to decipher the molecular basis of in meso crystallogenesis. Secondly, to automate and miniaturize the method and to make it more user-friendly and generally accessible. And, thirdly, to use the 
method to solve the structures of membrane proteins that are critical to human health. In what follows, the origins of the in meso method, its development as a high-throughput technique, the membrane proteins that have yielded to it, and the challenges ahead are described.

\section{The in meso method: how it works and the challenges it presents}

Setting up an in meso crystallization trial is straightforward. Typically, it involves combining two parts protein solution with three parts lipid at $20^{\circ} \mathrm{C}[11,12]$. The lipid most commonly used is the MAG (monoacylglycerol) mono-olein. According to the mono-oleinwater phase diagram (Figure 1) [13], and assuming there is no major influence on phase behaviour of the protein solution components, this mixing process should generate, by spontaneous self-assembly, the cubic mesophase (Figure 2) at, or close to, full hydration. The original method for mixing lipid and protein solution involved multiple cumbersome centrifugations in small glass tubes. Harvesting crystals required cutting the tubes and searching for small crystals through curved glass, which was not easy, and required experience, time and patience.

The cubic phase is sticky and viscous in the manner of thick toothpaste. As such, it is not easy to handle. In the course of our earlier lipid-phase science work, we had developed tools and procedures for manipulating such refractory materials. One of these, the syringe mixing device [14], was ideally suited to the task of combining microlitre volumes of monoolein with membrane protein solution in a way that produces protein-laden mesophase for direct use in crystallization trials with minimal waste. The mixer consists of two Hamilton microsyringes connected by a narrow bore coupler. Lipid is placed in one syringe, and protein solution in the other. Mixing is achieved by repeatedly moving the contents of the two syringes back and forth through the coupler [11]. The coupler is replaced by a needle for convenient dispensing of the homogeneous mesophase into wells of custom-designed glass sandwich crystallization plates $[15,16]$. Precipitant solutions of various compositions are placed over the mesophase and the wells are sealed with a coverglass. The plates are incubated at $20^{\circ} \mathrm{C}$ and monitored for crystal growth. Optical quality is the best it can be given that the mesophase is held between two glass plates and the mesophase itself is transparent. This means that crystals, just a few micrometres in size, can be seen readily using microscopy, whether or not the proteins are coloured. The use of cross-polarizers enhances the visibility of small crystals which usually appear birefringent in a dark background; the cubic phase itself is optically isotropic and non-birefringent. An added feature of the glass sandwich plates is that the double-sided tape used to create the wells provides almost hermetic sealing, ensuring minimal changes in well composition during the course of trials that can last for months. Step-by-step instructions, complete with an online video demonstration of the entire in meso crystallization process just described, have been published [11,12].

\section{The in meso robot}

The protocol just described refers to the manual mode of setting up crystallization trials. Accurate and precise delivery of the protein-laden mesophase in volumes that range from pico- to micro-litres was made possible by use of an inexpensive repeat dispenser in combination with differently sized microsyringes $[17,18]$. The smaller volumes means that the in meso method works with minuscule quantities of target protein. Thus extensive crystallization trials can be set up with just a few micrograms of valuable membrane protein, making the in meso method one of the most efficient in terms of protein requirement.

Whereas the repeat dispenser greatly facilitated the in meso method, it was still a manual set-up, with limits to the numbers of trials that any one person could comfortably set up at a 
sitting. The need to automate the process was obvious. With the assistance of A. Peddi and Y. Zheng, engineers at The Ohio State University where the original work was carried out, we were able to perform a proof-of-principle robotics exercise employing LabViewcontrolled motorized translation stages operating and supporting a microsyringe and a crystallization plate. With it, we demonstrated that the viscous mesophase could be dispensed automatically and wells were filled in such a way that eventually yielded crystals [19]. This was enough to secure funding for a robot which was custom-designed and built to our specifications.

The in meso robot has two arms programmed to move simultaneously. One dispenses the viscous protein-laden mesophase, while the other dispenses precipitant. Typical volumes used are $50 \mathrm{nl}$ of mesophase (consisting of $20 \mathrm{nl}$ of protein solution and $30 \mathrm{nl}$ of mono-olein) and $800 \mathrm{nl}$ of precipitant solution. Custom 96-well glass sandwich plates were designed which take approximately 6 min to fill using an eight-tip robot. The robot enables the precise and accurate setting up of in meso crystallization trials in high-throughput mode and, if required, under challenging conditions of reduced temperature and controlled lighting. Given the in meso robot's success, several are currently in use in laboratories throughout the world. A variant on the original design, where tip alignment is done automatically and where precipitant is handled by disposable tips, is now commercially available. It would appear to represent an important advance in simplifying the in meso set up and making the method user-friendly.

With the success that the in meso method has had, it perhaps is not unexpected to find products appearing on the market in support of this novel crystallogenesis approach. In addition to the in meso robots, these include a number of precipitant screen kits, glass and plastic sandwich plates, and a plate that comes complete with lipid-coated wells. The vendors indicate that the latter can be used with a liquid-dispensing robot for protein solution delivery first and precipitant post-swelling.

\section{Mesophase compatibility with protein solution components}

As alluded to above, what happens during in meso crystallization is intimately tied up with mesophase behaviour [20]. The working hypothesis for how nucleation comes about begins with the protein reconstituting into the continuous bilayer of the cubic phase (Figure 2). Precipitant is added which triggers local formation of a lamellar phase into which the protein preferentially partitions and concentrates in a process that leads to nucleation and crystal growth. Experimental evidence in support of aspects of this model has been reported $[20,21]$.

Experience built up over several years working with the in meso method suggests that the mesophase behaviour observed during the course of crystallization mimics that of the monoolein-water system (Figure 1). The implication therefore is that the protein solution has minimal effect on the phase behaviour of the hosting lipidic mesophase into which the protein is reconstituted. That solution, along with the target protein, typically includes lipid, detergent, buffers and salt at a minimum. Other components such as glycerol, thiol-groupspecific reagents or denaturants are not uncommon. Each of these can have an impact on phase behaviour and, by extension, on the outcome of a crystallization trial. In the interests of learning about component compatibility, the sensitivity of the mono-olein-water cubic phase system to their inclusion has been evaluated. Our findings indicate that the default cubic mesophase is remarkably resilient and retains its phase identity in the presence of a vast array of different additives. These include glycerolipids, cholesterol, free fatty acids, detergents, denaturants, glycerol and thiol-group-specific reagents [22-30]. Of course, for 
each there is a concentration beyond which the cubic phase is no longer stable nor, indeed, useful for crystallogenesis. In most cases, these limits have been identified.

Occasionally, the concentration of a protein solution component is not known exactly. Detergent is a case in point. This poses a problem because, if there is too much detergent, the bulk lamellar phase may form, but it will not support crystallization [22,31]. It may also be that a new detergent is being used whose compatibility with the cubic phase is not known. In this case, a small amount of the buffer used to solubilize the protein or the protein solution itself can be used to prepare mesophase. The physical texture, appearance between crossed polarizers, or SAXS (small-angle X-ray scattering) behaviour of the mesophase will indicate which phase has been accessed. If, for example, it is a lamellar phase that forms, suggesting too much detergent, then another purification step where its concentration in the final protein solution is reduced may be enough to solve the problem. We have encountered situations with bacteriorhodopsin where the particular preparation ended up having an excess of detergent. The mesophase first formed was lamellar, but, when it was used in combination with certain precipitants, a transition back to the cubic phase was induced which went on to support crystal growth [20].

\section{Screen solution compatibility}

As noted, in meso crystallization relies upon a bicontinuous mesophase which acts as a reservoir to feed protein into nucleation sites and for crystal growth (Figure 2). The crystallization screening process requires that chemical space be interrogated over wide limits to find conditions that support crystal growth. In the screening process the proteinladen mesophase is therefore typically exposed to precipitant solutions that encompass hundreds, perhaps even thousands, of different chemical compositions. Screen solution components typically include buffers that cover a wide $\mathrm{pH}$ range, polymers, salts, small organics, detergents, apolar solvents, amphiphiles, etc., and all at different concentrations. Each component can potentially destabilize the mesophase. In a separate study using SAXS, we examined the compatibility of the default mono-olein-water cubic phase with various commonly used precipitant screen solutions [25]. What we found was hardly surprising. Compatibility was temperature-dependent and the usual suspects, which included organic solvents, destroyed the cubic phase, rendering these screen solutions effectively useless. A goal of the study was to design screens that were mesophase-friendly. However, that goal was never pursued; instead, we have opted for the convenience of commercial screen kits mindful of the fact that certain conditions are not relevant. As a result, certain kits are simply not used because they contain too few conditions that are compatible with the cubic phase.

\section{Sponge phase}

During the course of mesophase-compatibility studies, we noticed that certain screen components caused the cubic phase to 'swell' and, under certain conditions, to form what is referred to as the sponge phase. The latter evolves from the cubic phase as a result of the 'spongifying' component lowering bilayer interfacial curvature, thereby enabling the mesophase to imbibe more lyotrope (aqueous solution). This is revealed in the SAXS pattern where the lattice parameter of the cubic phase rises. Eventually, the mesophase loses order and the low-angle diffraction pattern becomes diffuse. Fortunately, the sponge phase retains its bicontinuity and, as a result, can support in meso crystallogenesis [20,24,32]. One advantage of the sponge phase is that its aqueous channels are dilated. Thus proteins with large extramembrane domains should be accommodated in and amenable to crystallogenesis from the sponge phase. Furthermore, the reduced interfacial curvature is likely to facilitate more rapid and long-range diffusion within the lipid bilayer. Since net movement of protein 
from the bulk mesophase reservoir to the nucleation and growth sites is a requirement for crystallization, this effect alone should contribute to improved crystallization. Interestingly, many of the proteins that have yielded to the in meso method have been crystallized under conditions that favour sponge phase formation ([33] and see http://www.mpdb.tcd.ie).

Reflecting the utility of the sponge phase for in meso crystallogenesis, a number of commercial screening kits now include spongifiers such as poly(ethylene glycol), jeffamine and butanediol. Some of these provide a preformed sponge phase to which the protein solution is added directly. We continue to use the original method that involves an active protein reconstitution step and where the entire crystallization screen space is available for sampling.

\section{Rational lipid design for low-temperature crystallogenesis}

As described in the Introduction, the MS\&FB group has devoted considerable time and effort to establishing the rules for rationally designing lipids with specific end uses. One such application concerned the development of a host lipid for use in in meso crystallogenesis at low temperatures. Certain proteins are labile and require handling at low temperatures. The problem with the in meso method, in the default mode at least, is that it relies upon mono-olein as the hosting lipid. The cubic phase formed by mono-olein is not stable below $\sim 17^{\circ} \mathrm{C}[13]$ and performing crystallization trials in a cold room at $4-6^{\circ} \mathrm{C}$ is risky. For this low-temperature application a cis-mono-unsaturated MAG, 7.9 MAG, was therefore designed, using the rules referred to above. The target MAG was synthesized and purified in-house and its phase behaviour mapped out using SAXS [34]. As designed, it produced the cubic phase stable in the range $6-85^{\circ} \mathrm{C}$. $7.9 \mathrm{MAG}$ has been used in the crystallization of a number of membrane proteins by the MS\&FB group. The objective now is to make it, along with other synthetic MAGs (see below), available to the community by way of a commercial vendor.

The word 'risky' was used in the previous paragraph when referring to low-temperature crystallization with mono-olein as the hosting lipid. This reflects the fact that it is possible to do successful in meso work with mono-olein at $4^{\circ} \mathrm{C}$ provided the system undercools. Fortunately, the cubic phase is noted for this capacity $[13,35]$ and we regularly perform successful crystallization trials with mono-olein in the $4-17^{\circ} \mathrm{C}$ range. As expected, occasionally under these metastable conditions, the mesophase will convert into the lamellar crystalline or solid phase which is useless as far as crystallization is concerned.

Sugar-phytane lipids have been synthesized that form the fully hydrated cubic phase in the $10-70^{\circ} \mathrm{C}$ range [36] and that might find application for in meso crystallization at reduced temperatures.

\section{Additive lipid screening}

Early on in the development of the in meso method, I recognized that mono-olein, as the lipid used to create the hosting mesophase, is a most uncommon membrane lipid. The sense was that this MAG might rightly be regarded as foreign by certain target proteins and cause them to destabilize. One possible solution was to use a natural membrane lipid that would form the requisite cubic phase at $20^{\circ} \mathrm{C}$. None was available. An alternative was to use monoolein as the hosting lipid and to augment it with typical membrane lipids, thereby creating a more native-like environment. Accordingly, the carrying capacity of the monoolein cubic phase for a number of different lipids was established using SAXS [23]. This amounted to approximately $20 \mathrm{~mol} \%$ in the case of phosphatidylcholine,

phosphadidylethanolamine and cholesterol, with lesser amounts of phosphatidylserine and cardiolipin being accommodated. The approach of using additive lipids has had spectacular 
successes in the GPCR field where cholesterol doping of the cubic phase was critical to the production of structure-grade crystals [1,2,37-41].

\section{Host lipid screening}

Mono-olein was the first lipid used for in meso crystallogenesis. From the outset, it was recognized that this one lipid may not work with all target membrane proteins. These, in turn, come from a variety of native membranes which differ in lipid composition, surface charge and packing density, fluidity and polarity profile, bilayer thickness, intrinsic curvature, etc. Thus having a range of MAGs that differed in acyl chain characteristics with which to screen was deemed important. Using principles of rational design, a number of suitable MAGs were identified with the requirement that they form the cubic phase at $20^{\circ} \mathrm{C}$ under conditions of full hydration. A number of lipids meeting this specification have been synthesized and characterized in-house. They constitute a successful hosting lipid screen by the MS\&FB group. With several targets, that include $\beta$-barrels, $a$-helical proteins (see below) and an integral peptide antibiotic, crystals have been grown by the in meso method using these alternative MAGs $[34,42-44]$. In a number of cases, mono-olein either failed to produce crystals or the crystals it did produce were not of diffraction quality. It was only when MAGs from the hosting lipid screen were used that structure-grade crystals were obtained. The intent now is to make these novel MAGs available to the community through a suitable supplier.

\section{In meso structures}

At the time of writing, the in meso method accounts for almost 70 records in the PDB that relate to integralmembrane proteins and peptides ([33] and see http://www.mpdb.tcd.ie). This corresponds to $\sim 10 \%$ of all published membrane protein structures representing six distinct membrane protein types (Figure 3 ). With successes that include bacterial rhodopsins, light-harvesting complex II, photosynthetic reaction centres, $\beta$-barrels, GPCRs and an integralmembrane peptide, the method has a record of versatility and range. In the MS\&FB group, a multi-subunit cytochrome oxidase structure is in the final stages of refinement, which further attests to the generality of the method (J. Lyons, D. Aragão, T. Soulimane and M. Caffrey, unpublished work; PDB code 2YEV). Each of these membrane protein types represents bigger families, the members of which become suitable candidates for in meso crystallogenesis. The GPCR family is a case in point, with almost 800 distinct GPCRs coded for in the human genome alone. The in meso method, in combination with the necessary protein engineering and receptor stabilization strategies, is therefore now poised to contribute to the generation of GPCR structures in, what amounts to, production-line fashion. Evidence in support of this statement is the recent spate of receptor structures courtesy of the in meso method. We can only hope for the same degree of success with other membrane protein families.

The further development of the in meso crystallogenesis approach is an important goal for members of the MS\&FB group. One direction this has taken recently concerns the utility of the method with small membrane proteins. A separate analysis performed using a model cubic phase under restricted conditions indicated that suitable targets would need to include at least five transmembrane helices [45]. Our experience with the sponge-phase variant of the cubic phase suggested otherwise. Accordingly, the utility of the method with a 'miniprotein', the pentadecapeptide antibiotic linear gramicidin was investigated. It worked remarkably well, providing a structure with a resolution better than $1.1 \AA(1 \AA=0.1 \mathrm{~nm})$ $[44,46]$. This result is significant because it highlights the utility of the method with proteins having small transmembrane domains which abound in Nature. 


\section{The Membrane Protein Data Bank, statistics online}

Further details regarding the structure and function of integral, anchored and peripheral membrane proteins are available online in a convenient and searchable database, the MPDB (Membrane Protein Data Bank) at http://www.mpdb.tcd.ie [33]. Although records in the MPDB are obtained from the PDB, the former only includes entries for membrane proteins. Statistical analyses on the contents of the database can be performed and viewed online. Examples include detergents used for membrane protein structure work and the number of structures published annually by method.

\section{Prospects}

The in meso method burst on the scene a decade and a half ago. It was received with great anticipation for what it would deliver; perhaps it was to be the panacea. However, output in the early years was limited to naturally abundant bacterial a-helical proteins bedecked with stabilizing and highly coloured prosthetic groups ([33] and see http://www.mpdb.tcd.ie). The perceived restricted range, coupled to the challenges associated with handling the sticky and viscous cubic mesophase, meant that subsequent interest in the method waned. This was countered to some degree with the introduction of the in meso robot, a growing understanding for how the method worked at a molecular level, and a continued demonstration of the method's general applicability. However, interest in the method has rocketed of late, with the success that it has had in the GPCR field [1,2,37-41].

Improvements are, of course, needed if the method is to have longevity. Critically, the specialized materials and supplies upon which the method relies must be made more generally available and the method itself must be made user-friendly and routine. New and improved in meso robots available on the market are tackling the user-friendliness issue. Workshops that involve hands-on demonstrations contribute to making the method more accessible. I have been active in this area for several years now, with the latest workshop held as a part of the ICCBM13 meeting in Dublin in September 2010 (http:// www.iccbm13.ie) [47]. There, 36 students were trained in the practicalities and finer aspects of in meso crystallogenesis. A video demonstration of the method is available online [11].

Developments are needed in the area of crystal identification. Optical clarity is of the highest quality with the glass sandwich plates currently in use and this provides for ready detection of colourless micrometre-sized crystals in normal light and between crossed polarizers. Detection by UV fluorescence is particularly powerful and convenient for tryptophancontaining proteins. Fluorescence labelling [48] is also a route worth considering for the sensitive detection of early hits. SONICC (second-order non-linear optical imaging of chiral crystals) is a novel approach introduced by G. Simpson. It has been shown to sensitively and selectively detect membrane protein crystals growing in meso [49].

Recovering crystals from the mesophase for data collection is a non-trivial undertaking [12]. This is especially true when harvesting is done directly from glass sandwich plates. Typically, a glass cutter is used to open the well and to expose the mesophase. Teasing out and harvesting the crystal for immediate cryocooling is most conveniently done with a cryoloop. This is a slow, painstaking and cumbersome process especially if it must be done in a cold room and/or under subdued light. This whole area of harvesting calls out for innovation.

Data collection at the synchrotron is not exactly straightforward either. Given that in mesogrown crystals tend to be small, a mini-synchrotron X-ray beam is required. Oftentimes, the crystal of interest is hidden from view in a bolus of mesophase on the cryoloop. This means that locating the crystal and centring it requires rounds of diffraction rastering with a beam 
of progressively smaller size [50]. This same approach is used to advantage in finding the best diffracting part of a crystal. Locating crystals and centring based on X-ray fluorescence from heavy atoms in the sample is in development (D. Aragão, M. Becker, D. Li, M. Hilgart, J. Lyons, D. Yoder, S. Stepanov, R. Fischetti and M. Caffrey, unpublished work). Effective and efficient rastering is recognized now as an important feature of the latest MX beamlines at synchrotron facilities worldwide and steady improvements in the rastering process are being made. In situ screening and data collection are other areas under investigation.

The structures solved to date using in meso-grown crystals have relied on molecular replacement for phasing. However, new structures are in the offing that require experimental phasing. In our hands at least, this is proving to be a challenge. Several targets have been tackled using selenomethionine labelling and co-crystallization and soaking with heavy atoms without success. The problem derives from a low anomalous signal-to-noise ratio due to a combination of background scatter from adhering mesophase and the need to work with small and sometimes poorly diffracting radiation-sensitive crystals. This part of the in meso pipeline is in need of work.

Finally, the method should begin to be used with really big proteins and complexes. The sponge phase [24], with its open aqueous channels and flatter bilayer, should prove particularly useful in this regard. Using it in combination with novel hosting and additive lipid screens $[23,42]$ will go a long way towards producing crystals and ultimately highresolution structures where interactions that are integral to human health are revealed.

\section{Acknowledgments}

There are many who contributed to this work and most are from my own group, both past and present members. To all I extend my warmest thanks and appreciation.

Funding

This work was supported in part by Science Foundation Ireland [grant number 07/IN.1/B1836], Framework Programme 7 COST Action CM0902 and the National Institutes of Health [grant numbers GM75915, P50GM073210 and U54GM094599].

\section{Abbreviations}

GPCR G-protein-coupled receptor

MAG monoacylglycerol

MPDB Membrane Protein Data Bank

MS\&FB Membrane Structural and Functional Biology

MX macromolecular crystallography

SAXS small-angle X-ray scattering

\section{References}

1. Rasmussen SGF, Choi H-J, Fung JJ, Pardon E, Casarosa P, Chae PS, DeVree BT, Rosenbaum DM, Thian FS, Kobilka TS, et al. Structure of a nanobody-stabilized active state of the $\beta_{2}$ adrenoceptor. Nature. 2011; 469:175-180. [PubMed: 21228869]

2. Rosenbaum DM, Zhang C, Lyons JA, Holl R, Aragao D, Arlow DH, Rasmussen SGFR, Choi H-J, DeVree BT, Sunahara RK, et al. Structure and function of an irreversible agonist- $\beta_{2}$ andrenoreceptor complex. Nature. 2011; 469:236-240. [PubMed: 21228876] 
3. Warne A, Moukhametzianov R, Baker JG, Nehme R, Edwards PC, Leslie AGW, Schertler GFX, Tate CG. The structural basis for agonist and partial agonist action on a $\beta_{1}$-adrenergic receptor. Nature. 2011; 469:241-244. [PubMed: 21228877]

4. Caffrey M. J.D. Bernal and the genesis of structural biology. J. Physics Conf. Ser. 2007; 57:17-28.

5. Caffrey M, Kinsella JE. Growth and acyltransferase activity of rabbit mammary gland during pregnancy and lactation. J. Lipid Res. 1977; 18:44-52. [PubMed: 833507]

6. Caffrey M, Feigenson GW. Fluorescence quenching in model membranes: the relationship between $\mathrm{Ca}^{2+}$-ATPase enzyme activity and the affinity of the protein for phosphatidylcholines with different acyl chain characteristics. Biochemistry. 1981; 20:1949-1961. [PubMed: 6452902]

7. Caffrey M. Membrane protein crystallization. J. Struct. Biol. 2003; 142:108-132. [PubMed: 12718924]

8. Landau EM, Rosenbusch JP. Lipidic cubic phases: a novel concept for the crystallization of membrane proteins. Proc. Natl. Acad. Sci. U.S.A. 1996; 93:14532-14535. [PubMed: 8962086]

9. Caffrey M. A lipid's eye view of membrane protein crystallization in mesophases. Curr. Opin. Struct. Biol. 2000; 10:486-497. [PubMed: 10981640]

10. Nollert P, Qiu H, Caffrey M, Rosenbusch JP, Landau EM. Molecular mechanism for the crystallization of bacteriorhodopsin in lipidic cubic phases. FEBS Lett. 2001; 504:179-186. [PubMed: 11532451]

10a. Caffrey M. Crystallizing membrane proteins for structure determination: use of lipidic mesophases. Annu. Rev. Biophys. 2009; 38:29-51. [PubMed: 19086821]

11. Caffrey, M.; Porter, C. Crystallizing membrane proteins for structure determination using lipidic mesophases. J. Vis. Exp. 2010. http://www.jove.com/index/details.stp?id=1712

12. Caffrey M, Cherezov V. Crystallizing membrane proteins in lipidic mesophases. Nat. Protoc. 2009; 4:706-731. [PubMed: 19390528]

13. Qiu H, Caffrey M. Phase diagram of the monoolein/water system: metastability and equilibrium aspects. Biomaterials. 2000; 21:223-234. [PubMed: 10646938]

14. Cheng A, Hummel B, Qiu H, Caffrey M. A simple mechanical mixer for small viscous lipidcontaining samples. Chem. Phys. Lipids. 1998; 95:11-21. [PubMed: 9807807]

15. Cherezov V, Caffrey M. Nano-volume plates with excellent optical properties for fast, inexpensive crystallization screening of membrane proteins. J. Appl. Crystallogr. 2003; 36:1372-1377.

16. Cherezov V, Peddi A, Muthusubramaniam L, Zheng YF, Caffrey M. A robotic system for crystallizing membrane and soluble proteins in lipidic mesophasesActa Crystallogr. Sect. D Biol. Crystallogr. 2004; 60:1795-1807.

17. Cherezov V, Caffrey M. A simple and inexpensive nanoliter-volume dispenser for highly viscous materials used in membrane protein crystallization. J. Appl. Crystallogr. 2005; 38:398-400.

18. Cherezov V, Caffrey M. Picoliter-scale crystallization of membrane proteins. J. Appl. Crystallogr. 2006; 39:604-609.

19. Cherezov V, Peddi A, Muthusubramaniam L, Zheng YF, Caffrey M. A robotic system for crystallizing membrane and soluble proteins in lipidic mesophasesActa Crystallogr. Sect. D Biol. Crystallogr. 2004; 60:1795-1807.

20. Caffrey M. On the mechanism of membrane protein crystallization in lipidic mesophases. Cryst. Growth Des. 2008; 8:4244-4254.

21. Cherezov V, Caffrey M. Membrane protein crystallization in lipidic mesophases: a mechanism study using X-ray microdiffraction. Faraday Discuss. 2007; 136:188-205.

22. Ai X, Caffrey M. Membrane protein crystallization in lipidic mesophases: detergent effects. Biophys. J. 2000; 79:394-405. [PubMed: 10866965]

23. Cherezov V, Clogston J, Misquitta Y, Abdel-Gawad W, Caffrey M. Membrane protein crystallization in meso: lipid type-tailoring of the cubic phase. Biophys. J. 2002; 83:3393-3407. [PubMed: 12496106]

24. Cherezov V, Clogston J, Papiz M, Caffrey M. Room to move: crystallizing membrane proteins in swollen lipidic mesophases. J. Mol. Biol. 2006; 357:1605-1618. [PubMed: 16490208] 
25. Cherezov V, Fersi H, Caffrey M. Crystallization screens: compatibility with the lipidic cubic phase for in meso crystallization of membrane proteins. Biophys. J. 2001; 81:225-242. [PubMed: 11423409]

26. Clogston J, Caffrey M. Controlling release from the cubic phase: amino acids, peptides, proteins and nucleic acids. J. Controlled Release. 2005; 107:97-111.

27. Clogston J, Graciun G, Hart DJ, Caffrey M. Controlling release from the lipidic cubic phase by selective alkylation. J. Controlled Release. 2005; 102:441-461.

28. Liu W, Caffrey M. Gramicidin structure and disposition in highly curved membranes. J. Struct. Biol. 2005; 150:23-40. [PubMed: 15797727]

29. Liu W, Caffrey M. Interactions of tryptophan, tryptophan peptides and tryptophan alkyl esters at curved membrane interfaces. Biochemistry. 2006; 45:11713-11726. [PubMed: 17002272]

30. Cherezov V, Yamashita E, Liu W, Zhalnina M, Cramer WA, Caffrey M. In meso structure of the cobalamin transporter, BtuB, at $1.95 \AA$ resolution. J. Mol. Biol. 2006; 364:716-734. [PubMed: 17028020]

31. Misquitta Y, Caffrey M. Detergents destabilize the cubic phase of monoolein: implications for membrane protein crystallization. Biophys. J. 2003; 85:3084-3096. [PubMed: 14581209]

32. Wöhri AB, Johansson LC, Wadsten-Hindrichsen P, Wahlgren WY, Fischer G, Horsefield R, Katona G, Nyblom M, Oberg F, Young G, et al. A lipidic-sponge phase screen for membrane protein crystallization. Structure. 2008; 16:1003-1009. [PubMed: 18611373]

33. Raman P, Cherezov V, Caffrey M. The Membrane Protein Data Bank. Cell. Mol. Life Sci. 2006; 63:36-51. [PubMed: 16314922]

34. Misquitta Y, Cherezov V, Havas F, Patterson S, Mohan JM, Wells AJ, Hart DJ, Caffrey M. Rational design of lipid for membrane protein crystallization. J. Struct. Biol. 2004; 148:169-175. [PubMed: 15477097]

35. Briggs J, Caffrey M. The temperature-composition phase diagram of mono-myristolein in water: equilibrium and metastability aspects. Biophys. J. 1994; 66:573-587. [PubMed: 8011891]

36. Hato M, Minamikawa H, Salkar RA, Matsutani S. Phase behavior of phytanyl-chained akylglycoside/water systems. Prog. Colloid Polym. Sci. 2004; 123:56-60.

37. Rosenbaum DM, Cherezov V, Hanson MA, Rasmussen SG, Thian FS, Kobilka TS, Choi HJ, Yao XJ, Weis WI, Stevens RC, Kobilka BK. GPCR engineering yields high-resolution structural insights into $\beta_{2}$-adrenergic receptor function. Science. 2007; 318:1266-1273. [PubMed: 17962519]

38. Cherezov V, Rosenbaum DM, Hanson MA, Rasmussen SG, Thian FS, Kobilka TS, Choi HJ, Kuhn P, Weis WI, Kobilka BK, Stevens RC. High-resolution crystal structure of an engineered human $\beta_{2}$-adrenergic G protein-coupled receptor. Science. 2007; 318:1258-1265. [PubMed: 17962520]

39. Jaakola VP, Griffith MT, Hanson MA, Cherezov V, Chien EY, Lane JR, Ijzerman AP, Stevens RC. The 2.6 angstrom crystal structure of a human A2A adenosine receptor bound to an antagonist. Science. 2008; 322:1211-1217. [PubMed: 18832607]

40. Wu B, Chien EY, Mol CD, Fenalti G, Liu W, Katritch V, Abagyan R, Brooun A, Wells P, Bi FC, et al. Structures of the CXCR4 chemokine GPCR with small-molecule and cyclic peptide antagonists. Science. 2010; 330:1066-1071. [PubMed: 20929726]

41. Chien EY, Liu W, Zhao Q, Katritch V, Han GW, Hanson MA, Shi L, Newman AH, Javitch JA, Cherezov V, Stevens RC. Structure of the human dopamine D3 receptor in complex with a D2/D3 selective antagonist. Science. 2010; 330:1091-1095. [PubMed: 21097933]

42. Li D, Lee J, Caffrey M. Crystallizing membrane proteins in lipidic mesophases: a host lipid screen. Cryst. Growth Des. 2011; 11:530-537.

43. Misquitta LV, Misquitta Y, Cherezov V, Slattery O, Mohan JM, Hart D, Zhalnina M, Cramer WA, Caffrey M. Membrane protein crystallization in lipidic mesophases with tailored bilayers. Structure. 2004; 12:2113-2124. [PubMed: 15576026]

44. Hoefer N, Aragao D, Lyons JM, Caffrey M. Membrane protein crystallization in lipidic mesophases: hosting lipid effects on the crystallization and structure of a transmembrane peptide. Cryst. Growth Des. 2011

45. Grabe M, Neu J, Oster G, Nollert P. Protein interactions and membrane geometry. Biophys. J. 2003; 84:854-868. [PubMed: 12547769] 
46. Hofer N, Aragao D, Caffrey M. Crystallizing transmembrane peptides in lipidic mesophases. Biophys. J. 2010; 99:L23-L25. [PubMed: 20682243]

47. Pye V, Aragao D, Lyons JA, Caffrey M. Overview of the 13th International Conference on the Crystallization of Biological Macromolecules. Cryst. Growth Des. 2011 in the press.

48. Forsythe E, Achari A, Pusey ML. Trace fluorescent labeling for high-throughput crystallographyActa Crystallogr. Sect. D Biol. Crystallogr. 2006; 62:339-346.

49. Kissick DJ, Gualtieri EJ, Simpson GJ, Cherezov V. Nonlinear optical imaging of integral membrane proteins in lipidic mesophases. Anal. Chem. 2010; 82:491-497. [PubMed: 20025250]

50. Cherezov V, Hanson MA, Griffith MT, Hilgart MC, Sanishvili R, Nagarajan V, Stepanov S, Fischetti RF, Kuhn P, Stevens RC. Rastering strategy for screening and centring of microcrystal samples of human membrane proteins with a sub-10 microm size X-ray synchrotron beam. J. R. Soc. Interface. 2009; 6:S587-S597. [PubMed: 19535414] 


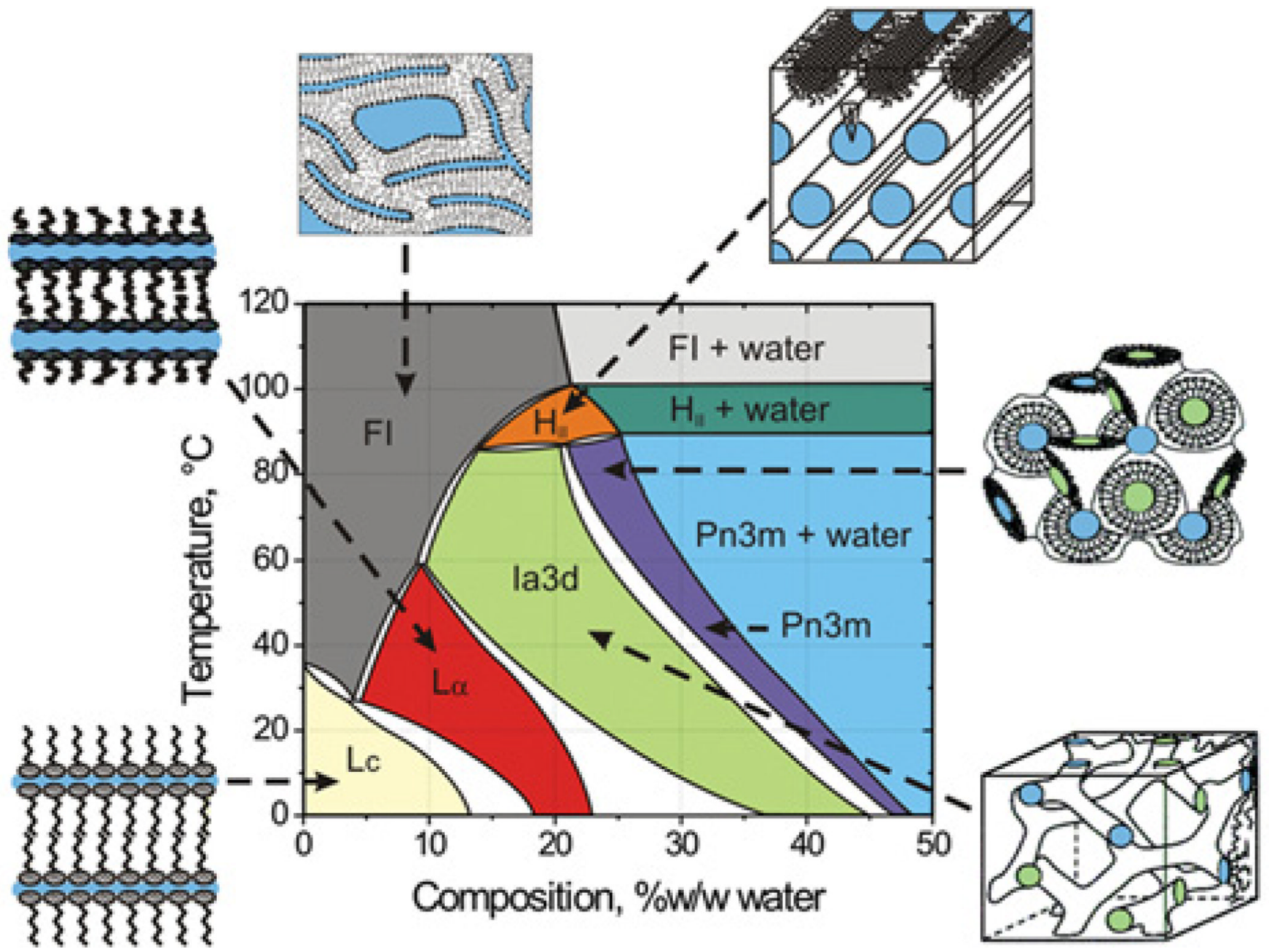

Figure 1. Temperature-composition phase diagram of the mono-olein-water system determined under 'conditions of use' in the heating and cooling directions from $20^{\circ} \mathrm{C}$

A cartoon representation of the various phase states is included in which coloured zones represent water. The liquid crystalline phases below $\sim 17^{\circ} \mathrm{C}$ are metastable [13].

Abbreviations: FI, fluid isotropic phase; $\mathrm{H}_{\mathrm{II}}$, inverted hexagonal phase; La, lamellar liquid crystalline phase; Lc, lamellar crystal phase. Figure reproduced from [24] with permission. 


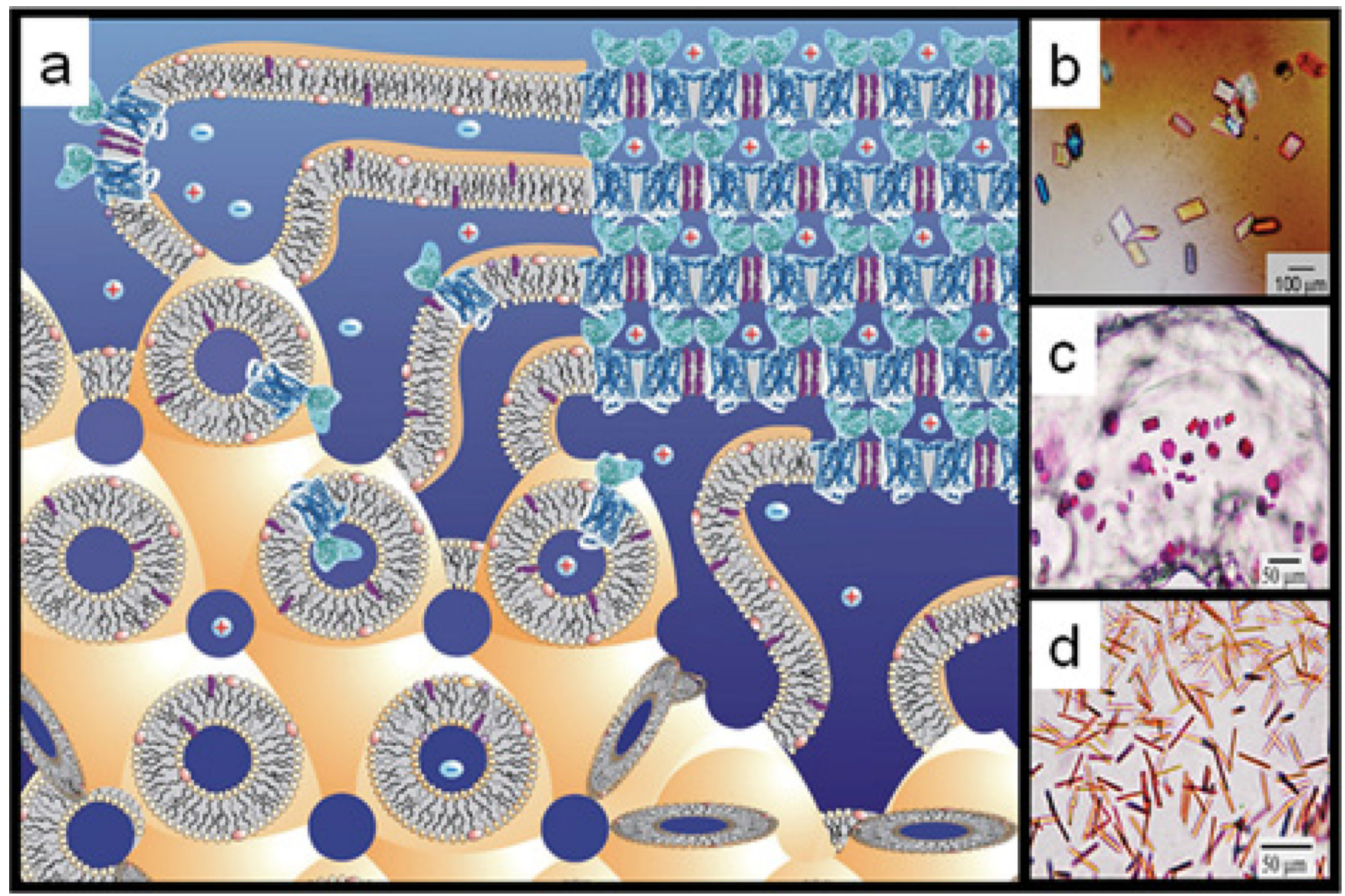

Figure 2. In meso crystallization model and crystals

(a) Schematic representation of the events proposed to take place during the crystallization of an integral membrane protein from the lipidic cubic mesophase. The process begins with the protein reconstituted into the highly curved bilayers of the bicontinuous cubic phase (bottom left quadrant). Added precipitants shift the equilibrium away from stability in the cubic membrane. This leads to phase separation, wherein protein molecules diffuse from the continuous bilayered reservoir of the cubic phase by way of a sheet-like or lamellar portal (upper left quadrant) to lock into the lattice of the advancing crystal face (upper right quadrant). Salt (positive and negative signs) facilitates crystallization, in part, by charge screening. Co-crystallization of the protein with native or added lipid (cholesterol) is shown in this illustration. As much as possible, the dimensions of the lipid (light yellow oval with tail), detergent (pink oval with tail), native membrane or added lipid (purple), protein (blue; $\beta_{2}$-adrenergic receptor-T4 lysozyme; PDB code 2RH1), and bilayer and aqueous channels (dark blue) have been drawn to scale. The lipid bilayer is approximately $40 \AA$ thick. Crystals of (b) BtuB, (c) bacteriorhodopsin and (d) light-harvesting complex II growing in meso. Reprinted, with permission, from the Annual Review of Biophysics [10a], Volume 38, () 2009 by Annual Reviews. 
(a) Gramicidin, B. brevis, $1.08 \hat{A}, 2 \Upsilon 2$
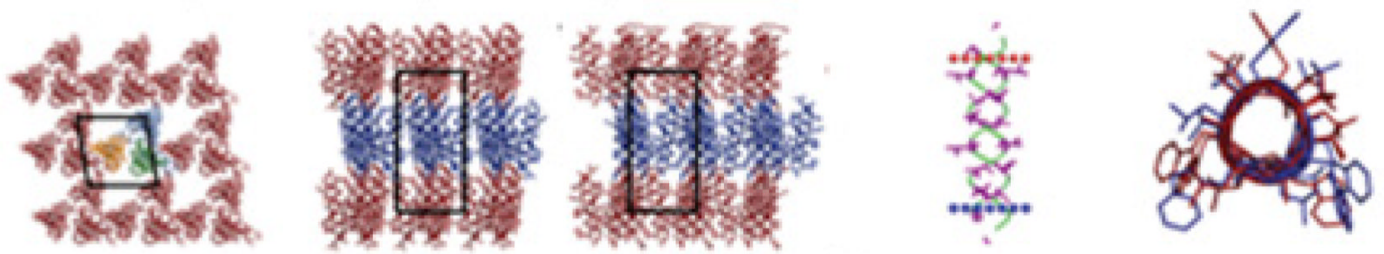

(b) Bacteriorhodopsin, H. salinanum, 1.43 $\AA, \underline{1 \mathrm{M} 0 \mathrm{~K}}$
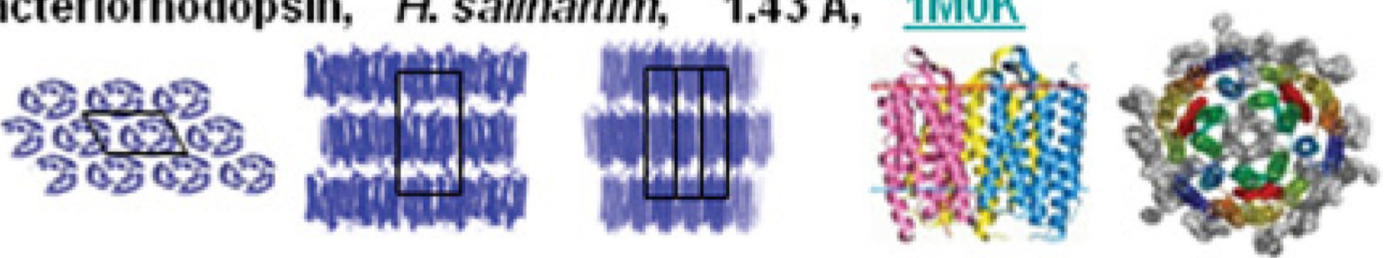

(c) Cobalamin transporter, E. coli, 1.95 $\AA$, 2GUF
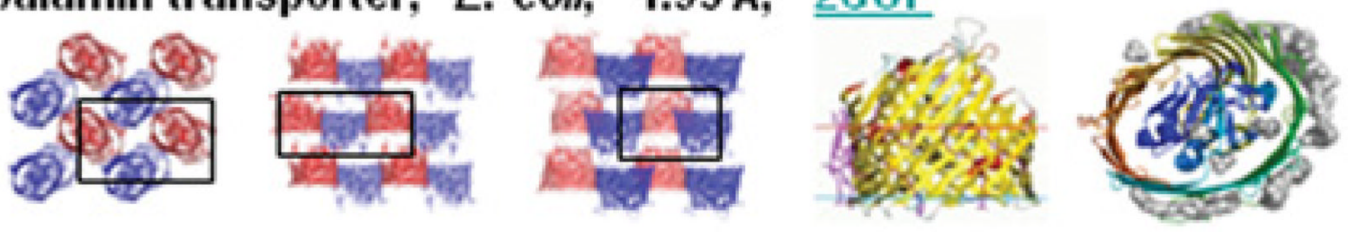

(d) Light harvesting complex II,
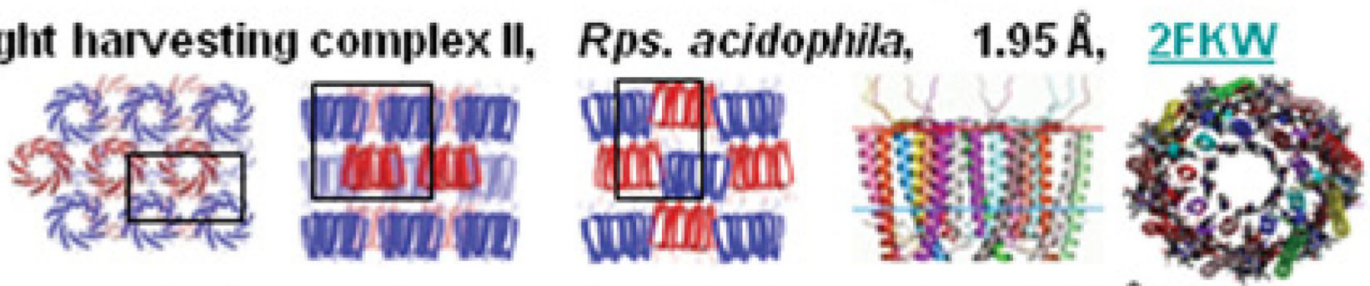

(e) Photosynthetic reaction center, $R$. sphaeroides, 2.20 $\AA, 2 \mathrm{GNU}$
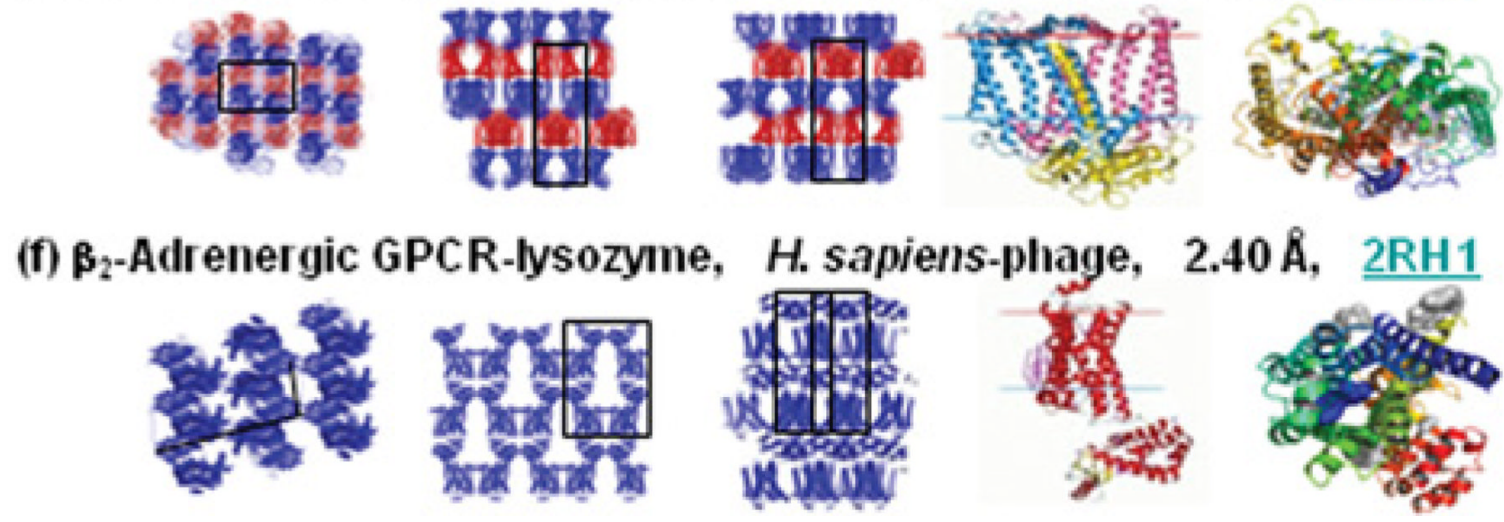

Figure 3.

In meso crystal packing arrangement and molecular structures of membrane proteins. The packing arrangement is shown in columns 1-3 within each panel. Expanded views of individual proteins or oligomers are shown in columns 4 and 5. The view in columns 1 and 5 represents a projection along the stacking axis. The views in columns $2-4$ are from within the plane of the stacked lamellae along the two other unit cell axes. In columns 1-3, black outlines are projections of the unit cell. Images in column 4 are from the Orientations of Proteins in Membranes (OPM) database (http://opm.phar.umich.edu/), in which the red and blue horizontal lines define the hydrophobic thickness of the protein. Proteins are identified by name, source organism, resolution and PDB code. Currently in meso records in the 
MPDB (http://www.mpdb.tcd.ie) number 44 for bacterial rhodopsins, six for the photosynthetic reaction centres, 13 for GPCR-T4 lysozyme chimaeras, three for gramicidin, two for $\beta$-barrels and one for light-harvesting complex II. The examples shown represent the highest resolution available for each of the six protein types [33] B. brevis, Bacillus brevis; H. salinarum, Halobacterium salinarum; E. coli, Escherichia coli; Rps. acidophila, Rhodopseudomonas acidophila; $R$. sphaeroides, Rhodobacter sphaeroides; H. sapiens, Homo sapiens. 